\title{
Modelling Text File Evaluation Processes
}

\author{
José Paulo Leal ${ }^{1}$ and Ricardo Queirós ${ }^{2}$ \\ ${ }^{1}$ CRACS \& INESC-Porto LA, Faculty of Sciences, University of Porto, \\ Rua do Campo Alegre, 1021 4169-007 Porto Portugal \\ zp@dcc. fc.up.pt \\ ${ }^{2}$ CRACS \& INESC-Porto LA, Faculty of Sciences, University of Porto, \\ Rua do Campo Alegre, 1021 4169-007 Porto Portugal \\ ricardo.queiros@eu.ipp.pt
}

\begin{abstract}
Text file evaluation is an emergent topic in e-learning that responds to the shortcomings of the assessment based on questions with predefined answers. Questions with predefined answers are formalized in languages such as IMS Question \& Test Interoperability Specification (QTI) and supported by many e-learning systems. Complex evaluation domains justify the development of specialized evaluators that participate in several business processes. The goal of this paper is to formalize the concept of a text file evaluation in the scope of the E-Framework - a service oriented framework for development of e-learning systems maintained by a community of practice. The contribution includes an abstract service type and a service usage model. The former describes the generic capabilities of a text file evaluation service. The later is a business process involving a set of services such as repositories of learning objects and learning management systems.
\end{abstract}

Keywords: e-learning, SOA, interoperability.

\section{Introduction}

The majority of e-learning systems include the automatic evaluation of quizzes as a feature. Quizzes have the advantage of being generic and usable in any learning domain. However, the most effective types of exercises in any learning domain, both for knowledge acquisition and for student grading, are seldom quizzes. For instance, it is hard to imagine learning computer programming without actually programming. An attempt to solve a programming exercise is written in a specific language (a programming language) that cannot be evaluated simply by comparing it with predefined answers, as in quiz evaluation.

Text file automatic evaluation differs significantly from quiz evaluation based on the IMS Question \& Test Interoperability (QTI) specification. QTI describes a data model for questions and test data and, since version 2.0, extends the IEEE Learning Object Metadata (LOM) standard with its own meta-data vocabulary. QTI was designed for questions with a set of pre-defined answers, such as multiple choice, multiple response, fill-in-the-blanks and short text questions. It supports also long text answers but the specification of their evaluation is outside the scope of the QTI. In 
fact, the evaluation of text files requires extra resources and specialized metadata. For this reason the authors consider that QTI is not adequate for text file automatic evaluation, as would be expected since it was not designed for this purpose. Extensions to learning object specification have to be developed to support text file evaluation [4]. Unlike text file evaluation, QTI quiz evaluation is integrated in many e-learning systems, especially in Learning Management Systems (LMS). On one hand text file evaluation is too specialized to justify its integration in a general LMS. On the other hand, provided as a service it can used by many kinds of systems. For instance, a programming evaluation service may have as clients programming assignment managers, self-evaluation tools and contest management systems. Its services can also be used by plug-ins of extensible systems, such as LMS or Integrated Development Environments (IDE).

The motivation for this research comes from the experience of the authors with systems such as Mooshak [5] and EduJudge [3]. The former is a contest management system for ICPC contests that is being used since 2002 also as an e-Learning tool in computer programming courses. The later is a system developed for enabling the access of LMS to the collection of programming exercises of the UVA on-line judge. Both systems include automatic evaluation components that if recast as services could provide their functions to different types of e-Learning systems.

The goal of this paper is to formalize services and processes involving text file evaluation in the scope of an e-learning framework. The purpose of an e-learning framework is to support the integration of systems within educational institutions using a Service Oriented Architecture (SOA) [1]. In this paper the authors report on the contribution to a particular e-learning framework - the e-Framework. This framework was selected based on a previous survey [2] since it has an active community of practice and accepts abstract definitions of services as contributions.

The contribution described in this paper includes an abstract definition of a type of service and a description of a business process model. The service modelled by the proposed definition receives a text file with an attempt to solve an exercise and produces an evaluation report. The exercise is referenced as a learning object (LO) available on an interoperable repository [3] supporting extended definitions of learning objects [4]. The business process model relates several abstract services definitions from the e-Framework, including the proposed service.

Examples of the applicability of this service usage model can be drawn from different areas, although the authors are particularly interested in the automatic evaluation of programming exercises. A program evaluation service compiles a program source code, executes it with test data and compares obtained and expected outputs contained in a learning object. Other examples of evaluator services process different types of text files: an electronic circuit evaluator receive a description of a circuit, injects input signals, simulates the circuit and compares output signals; a diagram evaluator receives a description of a diagram (e.g. UML) - a typed graph and tries to create a graph homomorphism with a solution. In all cases the service receives both a text file attempting to solve an exercise and a reference to an exercise specified as a learning object, containing other files with special roles in the evaluation process, and produces a detailed evaluation report. 